\title{
A new species of Ludovicius from China (Diptera: Dolichopodidae)
}

\author{
Lili Zhang \& Ding Yang*
}

Zhang, L. \& Yang, D. 2005: A new species of Ludovicius from China (Diptera: Dolichopodidae). - Entomol. Fennica 16: 305-308.

We review the species of the Ludovicius emeishanus-group from China, and describe one species as new to scicence, viz. Ludovicius songshanensis $\mathrm{sp} . \mathrm{n}$. We present a key to species of the group from China, and discuss the distribution of the Ludovicius emeishanus group in China.

Department of Entomology, China Agricultural University, Haidian, Beijing 100094, China; * correspondent author's e-mail: yangding@cau.edu.cn

Received 24 November 2003, accepted 28 January 2005

\section{Introduction}

The genus Ludovicius Rondani is characterized by the antennal scape rather thick but pedicel reduced and small, and arista usually one-segmented and nearly bare. The Palaearctic species of the genus Ludovicius were revised by Parent (1938) and Stackelberg (1941). Yang (1996a, b, 1998a-c, 1999a, b), Yang et al. (1998, 2002) and Yang \& Saigusa $(1999,2001)$ added 22 species to the fauna of China.

These 22 are mainly distributed in central and south-west China. Up to now, there are 12 known species from the Palaearctic Realm (Negrobov 1991, Grichanov 2000) and 15 known species from the Oriental Realm (Dyte 1975, Yang \& Saigusa 2001, Yang et al. 2002). Yang and Saigusa (2001) reviewed the Chinese species of the genus Ludovicius, which were classified in 8 species groups. In the present paper, the species of Ludovicius emeishanus-group from China are reviewed, and one species is described as new to science. The type specimens are deposited in the Insect Collection of China Agricultural University, Beijing.

The following abbreviations are used: acr acrostichal, ad anterodorsal, av anteroventral, dc dorsocentral, LI fore leg, LII mid leg, LIII hind leg, pd posterodorsal.

\section{The emeishanus-group}

Diagnosis. Thorax and abdomen at least partly yellow. Postocular bristles wholly black. Palpus yellow. Antenna partly or wholly yellow. acr absent or biseriate. Hind femur with 1 long ventral bristle at base. Male genitalia: cercus somewhat triangular with wide outer margin; hypandrium with slender lateral arm.

The emeishanus-group includes two species: Ludovicius emeishanus Yang and L. songshanensis sp. n.

\subsection{Key to species of emeishanus-group from China}

1. Thorax and abdomen partly yellow. Antennal scape black. acr absent. Sichuan (Emei Mountain) L. emeishanus Yang, 1998

- Thorax and abdomen yellow. Antenna wholly yellow. 6 pairs of acr. Beijing (Yanqing)

L. songshanensis sp. $\mathrm{n}$. 


\subsection{Ludovicius emeishanus Yang, 1998}

Ludovicius emeishanus Yang, 1998: 177.

Material examined. Holotype $\hat{\sigma}$, Sichuan: Emei Mountain (1,800-1,900 m a.s.1.), 25.VIII.1957, leg. Youca Yu.

Diagnosis. Thorax metallic green; pleuron yellow except pteropleuron blackish. Antennal scape black. acr absent. Abdomen yellow basally.

Distribution. China (Sichuan), Oriental Realm.

\section{Ludovicius songshanensis sp. n. (Fig. 1)}

Material examined. Holotype $\delta^{\lambda}$ : Beijing: Yanqing, Songshan Mountain (40 $51^{\prime} \mathrm{N}$, $115^{\circ} 79^{\prime} \mathrm{E} ; 785 \mathrm{~m}$ a.s.1.), 15.VII. 2003, leg. Dakang Zhou. Paratypes: Beijing: Yanqing, Songshan Mountain, $\left(40^{\circ} 51 \mathrm{~N}, 115^{\circ} 79 \mathrm{E}\right),(785$ m a.s.1.), 2 ふ઼َ, 15.VII.2003, leg. Chunfeng Jia.

Diagnosis. Thorax and abdomen yellow. Antenna yellow; arista dark brown, dorsal, with swollen apex. 6 pairs of acr. Legs yellow with mid and hind tarsi brownish from tip of tarsomere 1 onward. Cercus somewhat triangular with rather wide outer margin.

Description. Male. Head metallic green with pale gray pollen. Face narrower than first flagellomere. Hairs and bristles on head black; postocular bristles wholly black with posteroventral hairs yellow. Antenna (Fig. 1a) yellow, 2 times longer than wide; arista dark brown, dorsal, nearly bare, and with swollen apex. Proboscis brownish yellow with blackish hairs; palpus brownish yellow with blackish hairs.

Thorax yellow; mesonotum with 1 black lateral spot near wing base; lateropleuron with 1 black dorsal dot; pteropleuron with 1 black triangular dorsal dot. Hairs and bristles on thorax black; 5 dc, 6 pairs of acr; scutellum with 2 pairs of bristles (basal pair short and hair-like, apical pair long and strong); propleuron pale haired, with 1 pale bristle on lower part.

Legs yellow; mid and hind tarsi brownish from tip of tarsomere 1 onward. Hairs and bristles on legs black; mid and hind coxae each with 1 outer bristle; mid and hind femora each with 1 preapical bristle; hind femur with 1 pale thin bristle at base (longer than thickness of hind femur). Fore tibia with one row of short ad and 2 weak pd, apically with 3 short bristles; mid tibia with 2 ad,

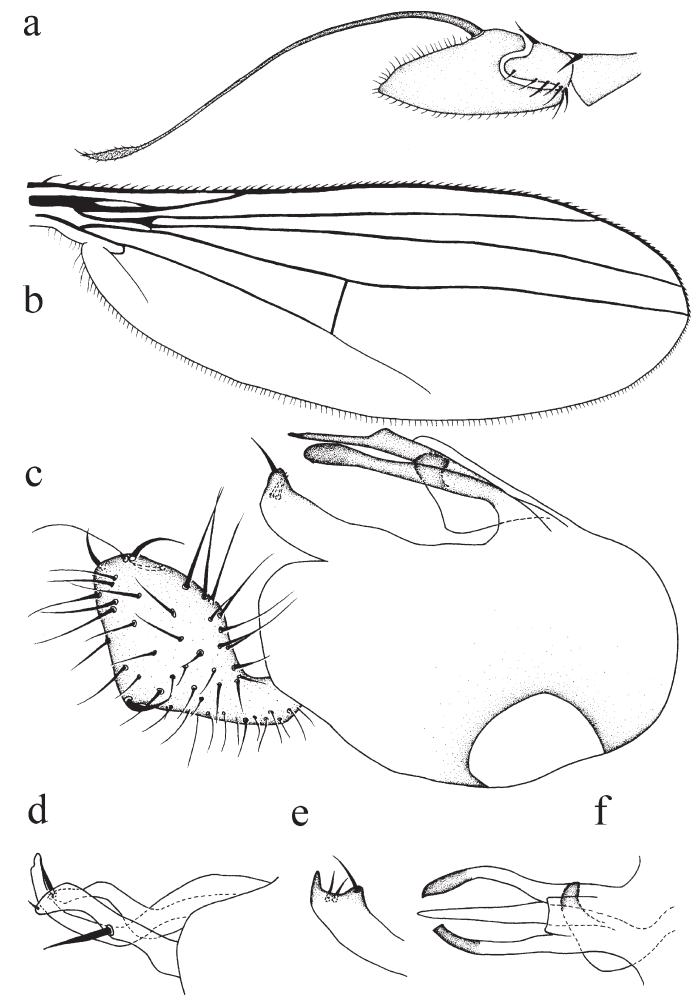

Fig. 1. Ludovicius songshanensis sp. n., male. a. Antenna, lateral view. b. Wing. c. Male genitalia, lateral view. d. Apical genital processes, lateral view. e. Lateral lobe on epandrium, posterior view. f. Hypandrium, ventral view.

2 pd and 1 av, apically with 4 bristles; hind tibia with 2 ad, 3 pd, apically with 3 bristles. Relative lengths of tibia and 5 tarsomeres: LI $1.0: 0.55$ : $0.3: 0.3: 0.2: 0.2 ;$ LII $1.75: 1.0: 0.5: 0.5: 0.3:$ 0.15 ; LIII $2.5: 0.55: 0.65: 0.5: 0.35: 0.2$.

Wing (Fig. 1b) hyaline, veins brown; $\mathrm{R}_{4+5}$ and $\mathrm{M}$ convergent apically, CuAx ratio 0.43. Squama yellow with blackish hairs. Halter yellow.

Abdomen yellow; hairs and bristles on abdomen black. Male genitalia (Fig. 1c-f): epandrium with long lateral lobe; cercus somewhat triangular with rather wide outer margin; hypandrium irregularly furcated.

Body length 3.3-3.4 mm, wing length 3.5-3.7 $\mathrm{mm}$.

Female. Unknown.

Distribution. China (Beijing), Palaearctic Realm.

Etymology. The specific name refers to the type locality Songshan Mountain. 
Fig 2. Distribution of Ludovicius emeishanus-group in China. Triangle $=$ Ludovicius emeishanus Yang; circle = Ludovicius songshanensis $\mathrm{sp}$. $\mathrm{n}$.

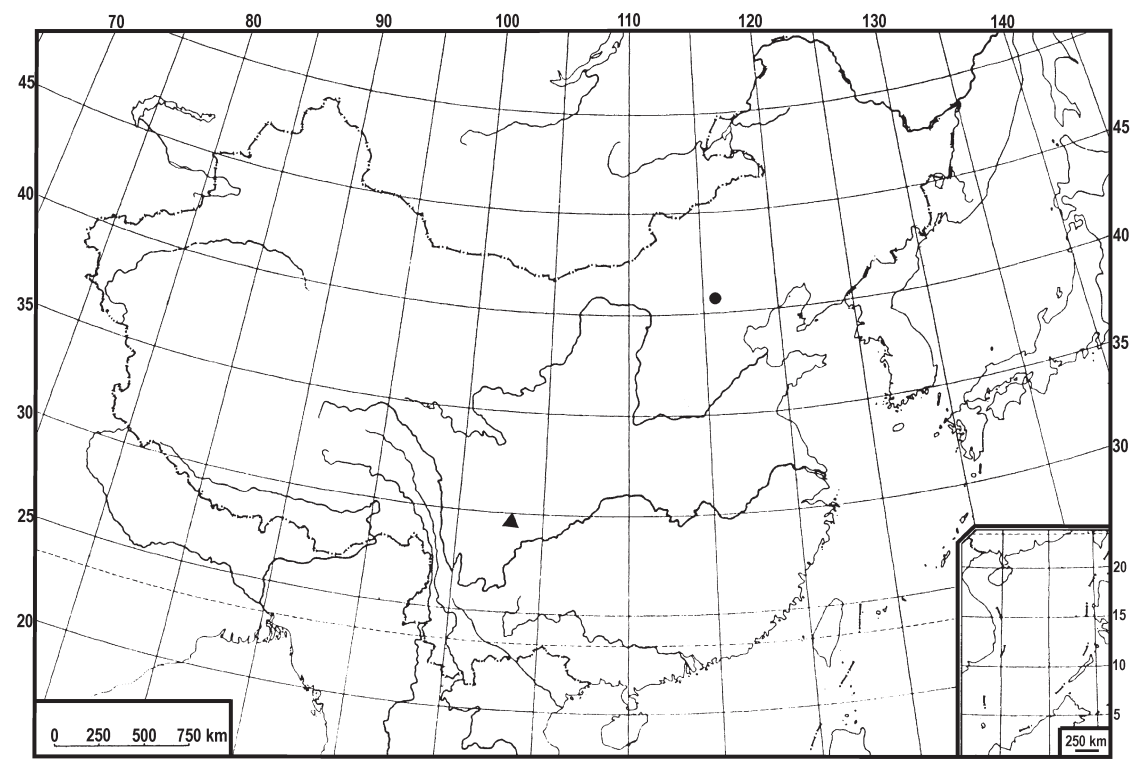

Differential diagnosis. This new species is similar to Ludovicius emeishanus Yang from Sichuan, but may be separated from the latter by the yellow thorax and abdomen and 6 pairs of acr. In L. emeishanus, thorax and abdomen are partly yellow, and acr is absent (Yang 1998).

\section{Discussion}

The species of emeishanus-group are scattered in Palaearctic and Oriental Realms. L. emeishanus Yang is distributed in South-west China Region (Southwest mountain Subregion, Sichuan). L. songshanensis $\mathrm{sp} . \mathrm{n}$. is distributed in North China Region (Loess plateau Subregion, Beijing). In fact, Ludovicius is a Palaearctic and Oriental genus that is highly diversified in Continental China, and further collecting is still needed to enrich the fauna of this genus.

Acknowledgements. We are grateful to D. K. Zhou and C. F. Jia for their specimens. The research was supported by National Natural Science Foundation of China (No. 30225009).

\section{References}

Grichanov, I. Ya. 2000: West-Palearctic species of the genus Ludovicius Rondani, 1843 (Diptera: Dolicho- podidae). — Russian Entomol. J. 9 (3): 269-274.

Negrobov, O. P. 1991: Family Dolichopodidae. - In: Soos, A. \& Papp, L. (eds.), Catalog of Palaearctic Diptera 7: 11-139. Akademiai Kiado Budapest.

Parent, O. 1926: Dolichopodides nouveaux de 1 xtreme orient palearctique. - Encycopedie Entomologique (B II), Diptera 3: 111-149.

Stackelberg, A. A. 1941: 29. Dolichopodidae. - In: Lindner, E. (ed.), Die Fliegen der Palaerktischen Region 4: 177-224. Schweitzerbart, Stuttgart.

Yang, D. 1996a: New species of Hercostomus and Ludovicius from North China (Diptera: Dolichopodidae). — Dtsch. entomol. Z. 43 (2): 235-244.

Yang, D. 1996b: Six new species of Dolichopodidae from China (Diptera, Dolichopodidae). — Bull. Inst. R. Sci. Nat. Belg. Ent. 66: 85-89.

Yang, D. 1998a: New and little known species of Dolichopodidae from China (III). — Bull. Inst. R. Sci. Nat. Nat. Belg. Ent. 68: 177-183.

Yang, D. 1998b: New species of Dolichopodidae from South China (Diptera, Dolichopodidae). Entomofauna 19: 233-240.

Yang, D. 1998c: Six new species of Dolichopodidae from China (Diptera). — Acta Entomol. Sin. 41 (Suppl.): 180-185.

Yang, D. 1999a: New and little known species of Dolichopodidae from China (IV). - Bull. Inst. R. Sci. Nat. Belg. Ent. 69: 197-214.

Yang, D. 1999b: Two new species of Dolichopodidae (Diptera) from North China. — Biologia 54(2): 165167.

Yang, D. 2001: A review of the Chinese species of the genus Ludovicius (Empidoidea, Dolichopodidae). Dtsch. Entomol. Z. 48(1): 83-92.

Yang, D., Grootaert, P. \& Song, H.Y. 2002: New and little known species of Dolichopodidae from China (XII). 
— Bull. Inst. R. Sci. Nat. Belg. Ent. 72: 213-220.

Yang, D. \& Saigusa, T. 1999: New species of Dolichopodidae from Henan (Diptera: Empidoidea). - In: Shen, X. \& Pei, H. (eds.), Insects of the Mountains Funiu and Dabie Regions, The fauna and taxonomy of Insects in Henan 4: 189-210. China Agricultural Scientech Press, Beijing.

Yang, D. \& Saigusa, T. 2001: New and little known species of Dolichopodidae (Diptera) from China (III). - Bull. Inst. R. Sci. Nat. Belg. Ent. 71: 165-188.

Yang, D., Yang, J. K. \& Li, Z. 1998: Three new species of Dolichopodidae from Henan (Diptera). - In: Shen, X. \& Shi, Z.Y. (eds.), Insects of the Mountains Funiu and Dabie Regions, The fauna and taxonomy of Insects in Henan 2: 81-84. China Agricultural Scientech Press, Beijing. 